face, showed no difference in the presence or absence of surface carboxyl groups, but when it incorporates the presence of the solvent, the calculations show the influence of the groups carboxyl in the interaction with the phenol molecule graphene surface. Thus, contrary to what is suggested in the literature, these results show that $\pi-\pi$ interactions do not play a decisive role in the adsorption mechanism.

\title{
Respuestas a plaguicidas y su relación con las interaccio- nes depredador-presa en anfibios (Amphibia, Anura) de agroecosistemas del Litoral Mesopotámico de Argentina
}

\section{Celina María Junges}

cmjunges@fbcb.unl.edu.ar

Dra. Paola Mariela Peltzer

Dr. Rafael Carlos Lajmanovich

Laboratorio de Ecotoxicología - Escuela Superior de Sanidad

Facultad de Bioquímica y Ciencias Biológicas

Universidad Nacional del Litoral

Fecha de la defensa: 19/03/2013

\section{Resumen}

En los últimos años, se ha puesto en evidencia que la presencia ambiental de plaguicidas está relacionada con efectos indirectos en los anfibios que se manifiestan a nivel de comunidad. Una explicación posible a este fenómeno podría atribuirse a que la incorporación de estos estresores en los ecosistemas no es la suficiente para causar la muerte fisiológica de los organismos, pero si capaz afectar su funcionamiento en un contexto ecológico, efecto conocido como muerte ecológica. Entre los distintos biomarcadores que se utilizan para detectar los efectos adversos de los plaguicidas sobre los anfibios se pueden citar las respuestas etológicas y las metabólicas.

El objetivo general de esta tesis fue analizar las interacciones depredador-presa entre distintas especies de larvas de anfibios y un pez depredador del litoral mesopotámico argentino, y establecer su relación con las variaciones en las respuestas etológicas (actividad natatoria y respiración) de las presas y metabólicas (actividad de las enzimas acetilcolinestera [AChE] y butirilcolinesterasa [BChE]) de depredador y presas en presencia de concentraciones subletales de tres plaguicidas: fenitrotion ( $F$ ), glifosato (Gly) y trifloxistrobin (TFS). Para la realización de las experiencias se seleccionó a Synbranchus marmoratus como depredador y a nueve especies de larvas de anfibios como presas: Rhinella arenarum (Bufonidae), Leptodactylus latrans, L. chaquensis (Leptodactylidae), Physalaemus santafecinus, $P$. albonotatus, $P$. riograndensis (Leiuperidae), Trachycephalus typhonius, Hypsiboas pulchellus (Hylidae) y Elachistocleis bicolor (Microhylidae). Bajo condiciones contraladas de laboratorio, se llevaron a cabo las experiencias que fueron organizadas en tres etapas: la primera de ecotoxicidad, la segunda de exposición, y la tercera de ensayos de depredación y evaluación de respuestas etológicas y metabólicas.

La primera etapa consistió en evaluar la toxicidad de las larvas de anfibios frente a for- 
mulaciones comerciales de los plaguicidas F, Gly y TFS, y establecer las concentraciones subletales de exposición a emplear en la evaluación de respuestas. En una segunda etapa, se realizó la exposición de depredador y presas a concentraciones subletales de los tres plaguicidas y a agua declorinada con la intención de clasificar a depredador y presas en dos tratamientos: expuestos a cada uno de los plaguicidas ( $F$, Gly y TFS) y no expuestos al plaguicida. Finalmente, tras la exposición de los organismos, se llevaron a cabo ensayos de depredación directa utilizando combinaciones de tratamientos que incluyeron depredador y presas expuestos y no expuestos a plaguicidas, sobre los cuales se evaluaron y contrastaron las respuestas etológicas y metabólicas.

La etapa de ecotoxicidad permitió demostrar que la toxicidad $\left(\mathrm{CL}_{50-24 h}\right)$ varió entre los agroquímicos evaluados, siendo más tóxico para las larvas de anfibios el fungicida TFS. En los ensayos de depredación directa, el tratamiento donde depredador y presas no fueron expuestos a plaguicidas (A-R-) presentó las mayores tasas de depredación, mientras que el tratamiento donde depredador y presas fueron simultáneamente expuestos a los plaguicidas $(A+R+)$ presentó las menores tasas de depredación. Estos resultados indican que la interacción depredador-presa fue alterada por un corto tiempo de exposición subletal a los plaguicidas F, Gly y TFS, y que además, dicha interacción fue sesgada en favor de las larvas de anfibios expuestas a los plaguicidas, ya que fueron significativamente menos depredadas por las anguilas. La reducción de la actividad natatoria y la respiración fueron las respuestas más observadas entre los renacuajos expuestos a los plaguicidas, hallándose una correlación positiva entre ambos parámetros. Las respuestas metabólicas fueron alteradas en sus niveles normales por la exposición a F, Gly y TFS, siendo la BChE más sensible como biomarcador que la AChE. En S. marmoratus, los niveles enzimáticos también respondieron a la exposición a los plaguicidas, alterando la actividad de la AChE y la BChE.

En conclusión, este estudio demostró que los plaguicidas F, Gly y TFS alteraron la interacción depredador-presa entre juveniles de S. marmoratus y larvas prometamórficas de anfibios del litoral mesopotámico argentino, interfiriendo sobre las respuestas etológicas de las presas y metabólicas de depredador y presas. Sin embargo, estas modificaciones no necesariamente pueden estar relacionadas con un aumento en la mortalidad de los individuos por depredación, ya que en este trabajo de tesis, el resultado final de la interacción depredador-presa fue sesgado en favor de las larvas de anfibios expuestas a los plaguicidas.

\section{Responses to pesticides and their relationship with predator-prey interactions in amphibians (Amphibia, Anura) from agroecosystems of Litoral Mesopotámico of Argentina}

\section{Summary}

In this doctoral thesis we proposed to analyze the predator-prey interaction between different species of tadpoles and a predator fish (Synbranchus marmoratus, ) and to establish their relationship with the variations in ethological responses from prey (swimming activity and bubbling) as well as metabolic ones (enzymatic activity of acetylcholinesterase [AChE] y butyrylcholinesterase [BChE]) from prey and predator, in the presence of sublethal concentrations of 
pesticides Fenitrothion (F), Glyphosate (Gly) and Trifloxystrobin (TFS).

In the direct predation test, the treatment where predator and prey were not exposed to pesticides (A-R-) exhibited the highest predation rates, while the treatment were predator and prey were simultaneously exposed to pesticides $(\mathrm{A}+\mathrm{R}+\mathrm{)}$ presented the lowest predation rates. These results indicate that predator-prey was biased in favor of the amphibians larvae exposed to pesticides since they were significantly less predated by the eels. The ethological responses were altered in tadpoles exposed to the pesticides, being the reduction of swimming activity and breath- ing the most frequent responses observed in pesticide-exposed tadpoles. The metabolic responses were altered in their normal levels by the exposition to F, Gly and TFS, being BChE more sensitive as biomarker than AChE when exposed to pesticides.

In conclusion, this study demonstrated that pesticides F, Gly and TFS altered the predator-prey interaction between $S$. marmoratus and tadpoles, interfering on preys' ethological and metabolic responses. However, these modifications cannot be necessarily related to an increase in mortality by predation, since the final result of this thesis was biased in favor of the amphibian larvae exposed to pesticides.

\section{Preparación de superficies de silicio poroso para el desarrollo de biosensores ópticos}

\author{
Liliana Carolina Lasave \\ llasave@intec.unl.edu.ar \\ Dr. Roberto Arce - Dra. Verónica González \\ Instituto de Desarrollo Tecnológico para la \\ Industria Química (INTEC) \\ Laboratorio de Semiconductores Nanoes- \\ tructurados \\ Facultad de Bioquímica y Ciencias Biológicas \\ Universidad Nacional del Litoral \\ Fecha de la defensa: 03/12/2013
}

\section{Resumen}

Esta tesis resume un estudio multidisciplinario realizado sobre el diseño y fabricación de dispositivos ópticos de silicio poroso nanoestructurado para aplicaciones en el campo de la detección bioquímica. El objetivo final de esta investigación está dirigido al desarrollo de biosensores libres de marcadores. Para ello se utilizan las propiedades ópticas de estos materiales en combinación con los efectos que sobre los mismos produce la incorporación de especies biológicas en su interior. El silicio poroso es un material ideal para la fabricación de transductores debido a su morfología de tipo esponja, caracterizada por un área de superficie específica de hasta $500 \mathrm{~m} 2 \mathrm{~cm}-3$, lo que asegura una interacción efectiva con gases y sustancias líquidas. Por otra parte, el silicio poroso es un material de bajo costo, totalmente compatible con los procesos microelectrónicos estándar.

Para este trabajo, se prepararon diferentes estructuras de silicio poroso, desde capas simples hasta multicapas (espejos de Bragg y microcavidades ópticas), las cuales se han caracterizado para su utilización 